\title{
Nivel de cumplimiento de información medioambiental en los reportes de sostenibilidad en sector minería y energía de empresas peruanas y chilenas
}

\section{Karina Pocomucha Valdivia, Cecilia Venegas Morales}

\author{
Facultad de Ciencias Económicas y Empresariales \\ Universidad de Piura \\ Facultad de Ciencias Económicas y Empresariales \\ Universidad de Piura
}

El objetivo del presente estudio es analizar el nivel de cumplimiento de la información medioambiental de acuerdo con la GRI G4 en los reportes de sostenibilidad de empresas de minería y energía de Perú y Chile. Se busca determinar si los factores tamaño de la empresa, cotización, exportación y naturaleza del capital influyen en el cumplimiento de dicha información. Este estudio se centra en la industria extractiva (minería, petróleo, gas y energía), ya que dichos sectores presentan los mayores problemas ambientales y sociales, y cubren la mayoría de los indicadores de sostenibilidad. Por lo tanto, el nivel de cumplimiento de información de responsabilidad social corporativa (RSC) por parte de la organización dependerá de las presiones sociales y políticas a las que se enfrente en relación con su desempeño medioambiental. Además, siguiendo la teoría de la legitimidad, se pretende conocer el grado de difusión de información ambiental. Los resultados indican que el $96 \%$ de las empresas divulgan información relacionada a mejoras y aspectos positivos con el fin de legitimarse. Asimismo, los factores tamaño y cotización ejercen una influencia significativa en el nivel de cumplimiento de información ambiental revelada en sus reportes.

Palabras clave: información ambiental, minería, energía, responsabilidad social corporativa

\section{Level of compliance with environmental information in the sustainability reports in the mining and energy sector of Peruvian and Chilean companies}

The objective of this study is to analyze the level of compliance with environmental information according to the GRI, G4 in the sustainability reports of mining and energy companies in Peru and Chile; to determine whether the factors size, price, export and nature of capital influence compliance with said information. This study focuses on the extractive industry (mining, oil, gas and energy), since these sectors present the greatest environmental and social problems and cover most sustainability indicators. Therefore, the level of CSR information compliance by the organization will depend on the social and political pressures it faces in relation to its environmental performance. In addition, it is intended to know following the theory of legitimacy, the degree of dissemination of environmental information. The results indicate that $96 \%$ of companies disclose information related to improvements and positive aspects to legitimize themselves; In addition, the size and price factors exert a significant influence on the level of compliance with environmental information disclosed in their reports.

Keywords: environmental information, mining, energy, corporate social responsibility 


\section{Nível de conformidade com informaçóes ambientais nos relatórios de sustentabilidade no setor de mine- ração e energia de empresas peruanas e chilenas}

O objetivo deste estudo é analisar o nível de conformidade com as informaçóes ambientais de acordo com a GRI, G4 nos relatórios de sustentabilidade de empresas de mineração e energia no Peru e no Chile; para determinar se os fatores tamanho, preço, exportação e natureza do capital influenciam o cumprimento dessas informaçóes. Este estudo se concentra na indústria extrativa (mineração, petróleo, gás e energia), uma vez que esses setores apresentam os maiores problemas ambientais e sociais e abrangem a maioria dos indicadores de sustentabilidade. Portanto, o nível de conformidade das informaçóes de RSC pela organização dependerá das pressốes sociais e políticas que enfrenta em relação ao seu desempenho ambiental. Além disso, pretende-se conhecer, seguindo a teoria da legitimidade, o grau de disseminação da informação ambiental. Os resultados indicam que 96\% das empresas divulgam informaçóes relacionadas a melhorias e aspectos positivos para se legitimar; Além disso, os fatores de tamanho e preço exercem uma influência significativa no nível de conformidade com as informaçóes ambientais divulgadas em seus relatórios.

Palavras-chave: informação ambiental, mineração, energia, responsabilidade social corporativa

\section{Introducción}

A lo largo de los años, los aspectos éticos y de sostenibilidad han ido ganando importancia en el desarrollo de las empresas. Por ello, los objetivos tradicionales, como maximizar las ventas y generar rentabilidad, se han abierto hacia la responsabilidad social (Vintró \& Comajuncosa, 2009). Esto es particularmente resaltante en las empresas del sector minero que tienen un gran impacto en la economía de Perú y Chile, países que son objeto de estudio del presente artículo. De hecho, en el año 2017, la minería representó en ambos casos alrededor del 10\% del PBI (Ministerio de Economía y Minas [MEM], 2017; Servicio Nacional de Geología y Minería [SERNAGEOMIN], 2017), y cerca del 62\% (MEM, 2017) y del 68\% (SERNAGEOMIN, 2017) del valor total de las exportaciones de Perú y Chile respectivamente.

Usualmente, la actividad minera presenta un elevado impacto tanto en el entorno (Jenkins, 2004; Loza, 2020) como en los niveles de la salud laboral de los trabajadores (Navarro \& Denis, 2003). Por ello, la presión que la sociedad ejerce sobre las empresas las obliga a adoptar códigos de conducta acordes con los valores ecológicos de los ciudadanos (Archel \& Lizárraga, 2001). Además, en ocasiones, el impacto ecológico ha devenido en conflictos ambientales y sociales que han impedido que las empresas obtengan una licencia social para su operación (Jenkins, 2004; Bebbington \& Bury, 2009). Todo esto se vincula con la rendición de cuentas (accountability), que implica la responsabilidad para llevar a cabo ciertas acciones y dar cuenta de ellas (Moneva, Archel, \& Correa, 2006; Gray, Owen, \& Adams, 1996; Rabasedas, 2018). Para las empresas mineras la accountability se basa en la rendición de cuentas de sus actividades a los accionistas, a los trabajadores, a las autoridades gubernamentales, a las comunidades campesinas y a la sociedad en general. Todos estos agentes ejercen presión haciendo hincapié en temas relacionados al impacto ambiental y social provocado por las actividades que emplean recursos naturales. En ese sentido, las labores y la situación de la empresa se deben dar a conocer por medio de los informes anuales y estados financieros (Venegas, 2016; Rabasedas, 2018). 
Las empresas se presentan por medio de sus estados financieros y reportes de sostenibilidad. En ellos, revelan su actividad empresarial, y dan a conocer su impacto económico, social y ambiental. Sin embargo, la preparación y divulgación de la información de sostenibilidad está escasamente regulada, pues, generalmente, tiene un carácter voluntario. Las empresas adoptan libremente las iniciativas de la Global Reporting Initiative (en adelante, GRI) o Pacto Mundial. En Perú, esto es promovido por asociaciones empresariales o sin fines de lucro. Entre ellas, se encuentra la Asociación Perú $2021^{1}$ (Hernández, 2016). Lamentablemente, no necesariamente la información que brindan las empresas voluntariamente es del todo completa: muchas buscarán legitimarse, por lo que solo divulgarán lo positivo o correcto de su actividad. Esto conlleva a deficiencias en la información (Venegas, 2016). De tal manera, se pierde el fin de servir de vehículo de comunicación para su evaluación por parte de los stakeholders (Moneva \& Llena, 2001).

Entre los medios más detallados y confiables para la comunicación de la información de las empresas, destaca la Guía para la Elaboración de Reportes de Sostenibilidad de la GRI (Daub, 2007; KPMG, 2011). KPMG (2011) corrobora que la misión de la GRI es crear un marco fiable y creíble para la elaboración de reportes de sostenibilidad, que pueda ser usado a nivel mundial. Debe tener parámetros comunes con el fin de tener criterios homogéneos fácilmente comparables que ayuden a los grupos de interés a entender el impacto que dichas organizaciones tienen en temas de sostenibilidad (Global Reporting Initiative [GRI], 2016). Por lo tanto, es muy importante el principio básico de la transparencia, dado que contribuye al aumento de la confianza de las organizaciones, la mejora de los resultados económicos y el aumento de la competitividad de las empresas (Bengua \& Clerici, 2009). Asimismo, influye positivamente en la medición y comparabilidad de los reportes de sostenibilidad (Aras \& Crowther, 2009; Unerman, Bebington, \& O’Dwyer, 2007).

En Latinoamérica, se puede afirmar que el análisis de reportes de memorias de sostenibilidad es una práctica comúnmente realizada en empresas del sector de minería, energía y finanzas (Alzate, Zapata \& Gómez, 2018; Del Barco, Del Barco \& Federici, 2018). El estudio de Hernández (2008), realizado sobre empresas del sector de minería en Perú, muestra la falta de uniformidad en las revelaciones de información económica, social y ambiental. Además, se indica que son pocas las empresas cotizadas que presentan reportes de sostenibilidad; las que lo hacen no presentan mucha información o no presentan información tan especializada.

Por otro lado, en las empresas peruanas, la publicación de información medioambiental en los reportes de sostenibilidad hace referencia, principalmente, a las prácticas de desempeño ambiental de acuerdo con la normativa vigente. Tal es el caso de las empresas mineras que informan sobre la implementación de Sistemas de Gestión de Calidad ISO 9001, Sistemas de Gestión Medioambiental ISO 14001 y Sistemas de Seguridad Laboral (Hernández, 2018). Además, revelan información referente al cuidado de los recursos naturales; al tratamiento de residuos; y a la gestión con las comunidades en temas ambientales y sociales. Estos aspectos pueden estar dirigidos a la búsqueda de legitimidad

\footnotetext{
Asociación civil sin fines de lucro, cuyo compromiso es la difusión y promoción de la responsabilidad social como una metodología de gestión empresarial, con el fin de impulsar el desarrollo sostenible del país
} 
(Gifford \& Kestler, 2008; Pajares, Morales \& Pintado, 2016; Venegas, 2016).

De Waal y Orcotoma (2011) indican que las empresas mineras multinacionales que aplican la responsabilidad social corporativa (en adelante, RSC) logran mejores resultados organizacionales que una institución que no la utiliza. Por lo tanto, es lógico proponer que la RSC no es solo una filosofía, sino que puede ser también una metodología adecuada para fines comerciales: una buena aplicación de la RSC podría ayudar a una organización a alcanzar alto rendimiento.

En la industria extractiva de Chile, los stakeholders perciben negativamente los impactos sociales y ambientales, en contraste con los impactos económicos, que son percibidos positivamente. La responsabilidad social empresarial (en adelante, RSE) se percibe como una campańa de marketing. En ese sentido, refleja un sentimiento anti trade-off, que puede usarse como una herramienta para compensar los impactos negativos de la minería (Viveros, 2016).

A partir de lo señalado anteriormente, se plantea como objetivo del presente estudio analizar el nivel de cumplimiento de información medioambiental en los reportes de sostenibilidad de empresas peruanas y chilenas publicados en la base de datos de la GRI en los periodos entre 2015 y 2017 pertenecientes a los sectores de minería y energía. Se busca verificar si existe una influencia de los factores tamaño, cotización, exportación y naturaleza del capital en el nivel de cumplimiento. Además de esta introducción, este estudio comprende una segunda parte, que presenta la revisión de la literatura y el marco teórico sobre la divulgación de información ambiental en reportes de sostenibilidad, así como el planteamiento de las hipótesis. La tercera parte explica el diseño de la investigación; y la cuarta, el análisis de los resultados para finalmente exponer las principales conclusiones.

\section{Revisión de literatura y marco teórico}

La RSC se define como el conjunto de acciones que toma una organización, con el objetivo final de asumir las responsabilidades derivadas del impacto de sus actividades sobre la sociedad y el medioambiente (Asociación Española para la Calidad [AEC], 2008). De acuerdo con la Asociación Española de Contabilidad y Administración de Empresas (AECA, 2004), "puede entenderse como el compromiso voluntario de las empresas con el desarrollo de la sociedad y la preservación del medio ambiente" (p. 9), y "su objetivo fundamental es introducir elementos de dirección y de gestión orientados a innovar y mejorar el impacto de las empresas, de forma que éstas generen externalidades socialmente responsables" (p.20).

Las bases teóricas para justificar las prácticas de sostenibilidad son diversas. Para la presente investigación se usará la teoría de la legitimidad (Warhurst, 2001; Husillos, 2007; Gray et al., 1996; Deegan \& Gordon, 1996; Da Silva \& Aibar, 2011; Ponce \& Labat, 2011; Asmeri, Alvionita, \& Gunardi, 2017; Corrigan, 2018). Esta considera que las empresas divulgan información sobre las acciones que realizan para reforzar su imagen y cambiar la percepción de la comunidad sobre ellas, con el objetivo de aumentar la legitimidad de su accionar y existencia (Venegas, 2016). En el marco de esta teoría, la divulgación de información medioambiental dependerá de las presiones sociales y políticas que ejerzan los stakeholders en relación a su desempeño medioambiental (Cho \& Patten, 2007; Kuruppu \& Milne, 2016; Venegas, 2016; Asmeri et al., 2017). Asimismo, les ayuda a garantizar la continuidad de su licencia social para seguir operando (Lodhia \& Hess, 2014; Kuruppu \& Milne, 2016; Böhling, Murguia \& Godfried, 2017).

La teoría de la legitimidad también puede ser entendida como aquel contrato social entre la empresa y la 
sociedad, en el que la sociedad será responsable del entorno donde opera la empresa (Ponce \& Labat, 2011; Deegan, 2002). En este sentido, cuando las empresas perciben que su legitimidad se ve amenazada por las preocupaciones del público respecto de los efectos de su actividad sobre el entorno, tienden a incrementar la divulgación de información medioambiental (Deegan \& Gordon, 1996; Brown \& Deegan, 1998; Wilmshurt \& Frost, 2000; O’Donovan, 2002; Cormier \& Gordon, 2001; Deegan, Rankin, \& Tobin, 2002; Ahmad \& Sulaiman, 2004; Branco, Eugénio, Ribeiro, Brammer, \& Pavelin, 2008; Cho, 2009; Venegas, 2016; Dube \& Maroun, 2017). La publicación de reportes de sostenibilidad antes era considerada una medida exclusiva de empresas proactivas. Sin embargo, con el paso de los ańos, se ha convertido en una práctica común (Moneva et al., 2006; Unerman et al., 2007; KPMG, 2011).

Por otro lado, el desempeño y la información de sostenibilidad de las empresas latinoamericanas se han producido de forma tardía con respecto a las empresas de países desarrollados. Esto se debe a que la adopción del modelo de la GRI se ha presentado prioritariamente en los sectores en los que los factores medioambientales y sociales ejercen un mayor impacto y representan mayores riesgos (Jenkins, 2004; Marimon, Alonso-Almeida, Rodríguez, \& Alejandro, 2012; Alonso-Almeida, Marimon, \& Llach, 2015; Hernández,2018). Con respecto a la industria extractiva (minería, petróleo, gas y energía), resulta importante analizar la RSC, pues se trata del sector con mayores problemas ambientales y sociales (Guenther, Hoppe, \& Poser, 2006; Dashwood, 2012; Park \& Ghauri, 2015; Alzate et al., 2018). Además, dicha industria cubre la mayoría de los indicadores de sostenibilidad (Peck \& Sinding, 2003; Guenther et al., 2006; Fonseca, McAllister, \& Fitzpatrick, 2014).

En Latinoamérica, la divulgación voluntaria de sostenibilidad corporativa ha crecido en la última década. Sin embargo, los estándares de información requeridos no han alcanzado elevados niveles de cumplimiento de la normativa internacional debido a una falta de políticas de sostenibilidad en las empresas y escasa influencia de entes reguladores públicos (Aranguren \& Maldonado, 2019; Del Barco et al., 2018; Rabasedas, 2018; García \& Hernández, 2019; Moneva, Jara-Sarrúa, Hernández, \& Del Barco, 2019; Loza, 2020). En Perú, las políticas de desarrollo económico y la exposición a mercados internacionales han impulsado a las empresas del sector minero a ampliar su desempeño no solo con los socios, sino también con otros grupos de interés, pues buscan establecer mejores relaciones con los distintos stakeholders (Quiroz, 2008; Nakasone, 2015; Hernández, 2016). Sin embargo, la mayoría de las empresas no han incorporado la responsabilidad corporativa en su gestión, ya que no es considerada como un aspecto estratégico y queda reducida a la búsqueda de una reputación social (Porro, 2008; Hernández, 2016) o de publicar lo "políticamente correcto" de su desempeño (Tostes \& Chero, 2010).

La proliferación de reportes de sostenibilidad en la industria extractiva ha sido objeto de muchos estudios en diferentes países, cuyo enfoque analítico ha sido predominantemente descriptivo (Guenther et al., 2006; Jenkins, 2004; Jenkins \& Yakovleva, 2006; Perez $\&$ Sanchez, 2009). Los estudios recientes analizan los siguientes aspectos: la mensurabilidad y comparabilidad entre empresas sobre el desempeño de sostenibilidad (Boiral \& Henri, 2017), la influencia del país de origen en las prácticas de RSE en empresas multinacionales (Buchanan \& Marques, 2018), el efecto de la rentabilidad y el desempeño ambiental en la divulgación de la RSE (Asmeri et al., 2017), el análisis de 
casos de empresas mineras (Dube \& Maroun, 2017; Böhling et al., 2017; Corrigan, 2018), el estado de la información ambiental como un elemento de la RSC (Guenther, 2006; Tost, Hitch, Chandurkar, Moser, \& Feiel, 2018), la contabilidad de la sostenibilidad (Lodhia \& Hess, 2014), la evaluación longitudinal de los reportes de sostenibilidad (Wirth, Kulczycka, Hausner, \& Koński, 2016; Perez \& Sánchez, 2009), y la evaluación de los patrones y la estructura de la información divulgada en RSE (Szczepankiewicz \& Mucko, 2016). Por otro lado, la mayoría de estudios parte de la teoría de la legitimidad (Ponce \& Labat, 2011; Lodhia \& Hess, 2014; Asmeri et al., 2017; Buchanan \& Marques, 2018; Böhling et al., 2017; Dube \& Maroun, 2017). Son pocos los que usan la teoría institucional (Corrigan, 2018), o una combinación de la teoría funcionalista, crítica y posmoderna (Boiral \& Henri, 2017).

Concretamente, en su análisis de empresas mineras, petroleras y de gas, el estudio de Guenther et al. (2006) demostró la influencia de las directrices GRI en los informes de sostenibilidad. En esa línea, concluye que la calidad de los informes de las empresas que siguen los estándares indicados por las guías GRI es superior a la de aquellas que no las siguen.

Entre los factores que influyen en el nivel de cumplimiento más utilizados por diferentes autores, se encuentra la variable tamaño de la empresa. Se trata de un aspecto muy importante en la divulgación de información medioambiental de sostenibilidad (Gray Kouhy, \& Lavers, 1995a; Deegan \& Gordon, 1996; Archel, 2003; Freedman \& Jaggi, 2005; Branco et al., 2008; Saleh, Zulkifli, \& Muhamad, 2010; Da Silva \& Aibar, 2011; Rabasedas, 2018), ya que actúa como un barómetro que explica por qué la empresa participa en la implementación de reportes de sostenibilidad (Saleh et al., 2010). En ese sentido, investigaciones previas muestran que el tamaño de la empresa tiene un efecto positivo y significativo (Djakman \& Machmud, 2008; Purwanto, 2011; Riantani \& Nurzamzam, 2015; Venegas, 2016). Siguiendo esa línea, para la medición, se ha considerado el número de trabajadores, ya que esta información se revela en los reportes de sostenibilidad (Hernández, 2018).

Se espera que las grandes empresas revelen más información porque enfrentan un mayor riesgo y presión política. Por tanto, una mayor divulgación de sostenibilidad es el esfuerzo realizado por la empresa para reducir los costos políticos y evitar gastos elevados debido a las demandas de la sociedad (Sembiring, 2005). Por otro lado, las empresas de menor tamaño usualmente disponen de menos recursos para suministrar dicha información y suelen tener una posición reacia a divulgarla, pues estarían suministrando datos confidenciales que afectarían la posición competitiva de la compañía (Da Silva \& Aibar, 2011; Venegas, 2016).

La nacionalidad de la empresa, y el país en el que opera y difunde su información pueden también condicionar el contenido y extensión de los datos divulgados. Esto se debe a las diferentes normativas legales en materia medioambiental o a una cultura medioambiental más arraigada en la sociedad (Da Silva \& Aibar, 2011). Además, una significativa participación de capitales extranjeros en la financiación de la empresa parece ejercer cierta influencia en la divulgación de información de sostenibilidad corporativa. Se observa las empresas adoptan los estándares de los países con mayor cultura medioambiental en los que se instalan (Gray, Kouhy, \& Lavers, 1995b; Archel \& Lizarraga, 2001; Venegas, 2016; Cormier \& Magnan, 2003). Por eso, si las empresas cuentan con un gran porcentaje de accionistas extranjeros, tenderán a divulgar más información ambiental en sus reportes 
de sostenibilidad: resulta más difícil para ellos obtener información acerca de la compañía por fuentes alternativas y lo que se pretende es reducir la asimetría de información.

Algunos estudios concluyen que hay una relación positiva entre el hecho de cotizar en mercados organizados y el nivel de información de responsabilidad social revelada (Cooke, 1989; Meek, Roberts \& Gray, 1995; Leuz, 2000; Ponce \& Labat, 2011). Esto se debe a que las empresas que cotizan en bolsa contarán con un mayor número de usuarios de sus estados financieros, los mismos que exigirán una mayor información distinta a la obligatoria (Ponce \& Labat, 2011). Por su parte, Williams (1999) señala la influencia de los factores culturales y sociales del país de origen en la extensión de la divulgación de información medioambiental de empresas cotizadas (citado en Venegas, 2016, p. 150). Además, Freedman y Jaggi (2005) comprueban la influencia de la cultura medioambiental del país de origen de la matriz en el nivel de información revelada por las filiales que operan en países menos activos o sensibles en términos de protección medioambiental. En lo que respecta a las empresas latinoamericanas, las que han tenido un mayor desarrollo y adopción de las prácticas de desempeño e información de sostenibilidad han sido las subsidiarias de grupos transnacionales y las empresas que exportan a países desarrollados (Islam \& Deegan, 2010; Momin \& Parker, 2013; Hernández, 2018). Finalmente, cabe resaltar que, a partir del mes de julio de 2018, las empresas que decidan seguir divulgando sus reportes de sostenibilidad deberán atenerse a los nuevos estándares elaborados por la GRI, que presentan una estructura basada en módulos interrelacionados (Del Barco et al., 2018).

A partir de la información expuesta, se plantean las siguientes hipótesis para la presente investigación:
- H1: Determinar si el factor tamaño influye en el nivel de cumplimiento de los reportes de sostenibilidad de empresas del sector de minería y energía de Perú y Chile

- H2: Determinar si el factor naturaleza del capital influye en el nivel de cumplimiento de los reportes de sostenibilidad de empresas del sector de minería y energía de Perú y Chile

- H3: Determinar si el factor cotización influye en el nivel de cumplimiento de los reportes de sostenibilidad de empresas del sector de minería y energía de Perú y Chile

- H4: Determinar si el factor exportación influye en el nivel de cumplimiento de los reportes de sostenibilidad de empresas del sector de minería y energía de Perú y Chile

\section{Metodología}

Esta investigación comparativa es de naturaleza cuantitativa con un alcance descriptivo correlacional. La investigación consiste en un análisis transversal de los reportes de sostenibilidad de los periodos entre 2015 y 2017. Se trata de los años más recientes que se encuentran disponibles a la fecha de la investigación. Además, se ha aplicado la metodología de análisis de contenido de reportes de sostenibilidad, usada en estudios como los de Gray et al. (1995b), Moneva, Acero y Llena (2007), Sweeney y Coughlan (2008), Szczepankiewicz y Mucko (2016), Hernández (2016), Boiral y Henri (2017), Rabasedas (2018), y Del Barco et al. (2018).

Para medir el nivel de cumplimiento en la información medioambiental revelada, se ha usado la GRI G4. La información se agrupó de acuerdo a las siguientes categorías: materiales, energía, agua, biodiversidad,

Contabilidad y Negocios (16) 31, 2021 / e-ISSN 2221-724X 
emisiones, efluentes y residuos, cumplimiento regulatorio, transporte, aspectos generales, evaluación ambiental de los proveedores, y mecanismos de reclamación ambiental. Las categorías fueron medidas en 52 variables. Además, se utilizaron las siguientes escalas de ponderación: 1) "informado parcialmente" (valor 1) si se encontró al menos uno de los componentes requeridos en el informe, 2) "informado totalmente" (valor 2) si se encontró todo lo requerido en la GRI G4 y 3) "no informado" (valor 0) si no aplica (valor 3). Estas categorías coinciden con las usadas por Guenther et al. (2016). La metodología de ponderación del cumplimiento de determinados criterios (Warsame, Simmons, \& Neu, 2002; Freedman \& Jaggi; 2005; Van Staden \& Hooks, 2007) es una de las más utilizadas en investigaciones de RSC (Gray et al., 1995a), ya que muestra una descripción del estado y el nivel de cumplimiento de la divulgación medioambiental en las empresas (Mathews, 1997).

Para la medición de la variable independiente tamaño de la empresa, se ha considerado el número de trabajadores, ya que esta información se revela en los reportes de sostenibilidad (Ponce \& Labat, 2011; Hernández, 2018). Además, se considera que las empresas con una mayor cantidad de empleados tendrán mayores demandas de información por parte de estos (Ponce $\&$ Labat, 2011). Por ello, para la presente investigación, se ha considerado el logaritmo neperiano del número de trabajadores.

Para evaluar la naturaleza del capital, se ha considerado si es extranjero, nacional o mixto. Con respecto al factor cotización y exportación (se ha codificado con "valor 0" el no; y con "valor 1", el sí), la clasificación de los sectores elegidos se ha realizado sobre la base de la división de la GRI "minería” y "energía"2.

Con respecto a las técnicas de análisis estadístico utilizadas, se aplicó un análisis de regresión lineal múltiple para evaluar la influencia de las variables seleccionadas y las comparaciones de medias mediante ANOVA. Para la muestra, se han considerado las empresas pertenecientes al sector de minería y energía de Perú y Chile que publicaron sus reportes de sostenibilidad en la base de datos de la GRI en el transcurso de los periodos entre 2015 y 2017. En total, eran 44 empresas. Sin embargo, no todas presentaron información de los tres periodos analizados. En total, se obtuvieron 80 reportes de sostenibilidad, detallados en la tabla 1 .

El sector de minería ha sido seleccionado porque existe una amplia gama de problemas de desarrollo sostenible en dicha actividad. Por este motivo, la mayoría de empresas del sector utilizan el informe GRI (Guenther et al., 2006; Perez \& Sánchez, 2009; Fonseca et al., 2014; Nakasone, 2015; Szczepankiewicz \& Mucko, 2016; Boiral \& Henri, 2017; Asmeri et al., 2017; Tost et al., 2018; Alzate et al., 2018). Además, las actividades de los dos sectores seleccionados presentan un gran potencial de causar daño ambiental, que puede afectar a las comunidades circundantes. Por eso, es muy importante que las empresas comprendan e implementen el concepto de responsabilidad corporativa para evitar conflictos potenciales entre las comunidades locales (Jenkins, 2004; Jenkins \& Yakovleva, 2006; Dong \& Xu, 2016; Szczepankiewicz \& Mucko, 2016).

2 Cabe resaltar que en este rubro hay empresas de gas y petróleo. 
Tabla 1. Reportes de sostenibilidad de empresas peruanas y chilenas (2015-2017)

\begin{tabular}{|c|c|c|c|c|c|}
\hline Empresa & País & Sector & 2015 & 2016 & 2017 \\
\hline Antamina & Perú & Minería & GRI G4 & GRI G4 & 0 \\
\hline Antapaccay & Perú & Minería & 0 & GRI G4 & 0 \\
\hline Buenaventura & Perú & Minería & 0 & Non-GRI & Non-GRI \\
\hline Compañía Minera Ares & Perú & Minería & 0 & 0 & 0 \\
\hline Compañía Minera Poderosa S.A. & Perú & Minería & GRI G4 & Citing GRI & Citing GRI \\
\hline El Brocal & Perú & Minería & GRI G4 & GRI G4 & Non-GRI \\
\hline GOLD FIELDS EN PERÚ & Perú & Minería & GRI G4 & GRI G4 & 0 \\
\hline LAS BAMBAS & Perú & Minería & GRI G4 & GRI G4 & 0 \\
\hline Milpo & Perú & Minería & GRI G4 & GRI G4 & 0 \\
\hline Minsur & Perú & Minería & GRI G4 & GRI G4 & 0 \\
\hline VOLCAN CIA MINERA S.A.A. & Perú & Minería & GRI G4 & GRI G4 & 0 \\
\hline Yanacocha & Perú & Minería & GRI G4 & GRI G4 & GRI G4 \\
\hline Anglo American Chile & Chile & Minería & GRI G4 & GRI G4 & \\
\hline Antofagasta Minerals & Chile & Minería & GRI G4 & GRI G4 & GRI Standards \\
\hline BHP Billiton Chile & Chile & Minería & Citing GRI & Citing GRI & 0 \\
\hline GRUPO CAP & Chile & Minería & GRI G4 & 0 & 0 \\
\hline CAP ACERO & Chile & Minería & 0 & Non-GRI & 0 \\
\hline CAP MINERÍA & Chile & Minería & GRI G4 & 0 & 0 \\
\hline Codelco & Chile & Minería & GRI G4 & GRI G4 & GRI Standards \\
\hline Compañía Minera Doña Inés de Collahuasi & Chile & Minería & GRI G4 & GRI G4 & 0 \\
\hline Ecometales & Chile & Minería & Citing GRI & Citing GRI & Citing GRI \\
\hline Empresa Nacional de Minería (ENAMI) & Chile & Minería & GRI G4 & GRI G4 & GRI G4 \\
\hline Minera Valle Central & Chile & Minería & 0 & 0 & GRI G4 \\
\hline Chilectra & Chile & Energía & GRI G4 & 0 & 0 \\
\hline Colbún & Chile & Energía & GRI G4 & GRI G4 & GRI Standards \\
\hline Empresa Nacional de Electricidad (Endesa Chile) & Chile & Energía & GRI G4 & GRI G4 & 0 \\
\hline Enel Chile S.A. & Chile & Energía & 0 & GRI G4 & 0 \\
\hline Enel Generación Chile S.A. & Chile & Energía & 0 & GRI G4 & 0 \\
\hline Enersis & Chile & Energía & GRI G4 & 0 & 0 \\
\hline GNL Quintero & Chile & Energía & GRI G4 & 0 & 0 \\
\hline Transelec & Chile & Energía & GRI G4 & GRI G4 & GRI Standards \\
\hline
\end{tabular}




\begin{tabular}{|l|c|c|c|c|c|}
\hline \multicolumn{1}{|c|}{ Empresa } & País & Sector & 2015 & 2016 & 2017 \\
\hline CELEPSA Perú & Perú & Energía & 0 & GRI G4 & 0 \\
\hline CENERGIA & Perú & Energía & 0 & 0 & GRI Standards \\
\hline Consorcio Transmantaro & Perú & Energía & & GRI G4 & \\
\hline Contugas & Perú & Energía & GRI G4 & GRI G4 & GRI Standards \\
\hline Duke Energy Perú & Perú & Energía & GRI G4 & GRI G4 & 0 \\
\hline Electroperu & Perú & Energía & GRI G4 & GRI G4 & 0 \\
\hline Electro Sur Este & Perú & Energía & 0 & GRI G4 & 0 \\
\hline Enel Generación Piura & Perú & Energía & 0 & 0 & GRI Standards \\
\hline ESSOLIN & Perú & Energía & 0 & 0 & GRI Standards \\
\hline PETROPERÚ & Perú & Energía & GRI G4 & GRI G4 & 0 \\
\hline Repsol Perú & Perú & Energía & GRI G4 & GRI G4 & GRI G4 \\
\hline SATEL PERU & Perú & Energía & 0 & 0 & GRI Standards \\
\hline TGP & Perú & Energía & Non-GRI & Non-GRI & Non-GRI \\
\hline
\end{tabular}

\section{Análisis de resultados}

La tabla 2 muestra la cantidad de reportes de sostenibilidad de empresas latinoamericanas pertenecientes a los sectores de minería y energía que han sido publicados en la base de datos de la GRI. Se puede observar que Perú es el tercer país en orden de número de reportes de sostenibilidad publicados en los periodos entre 2015 y 2017. Por su parte, Chile ocupa el cuarto lugar. Cabe resaltar que la frecuencia de publicación de las empresas no ha sido periódica.

La figura 1 nos permite hacer el análisis por país. Muestra que la tendencia en los periodos entre 2015 y 2017 en las empresas chilenas consiste en disminuir las publicaciones de sus reportes de sostenibilidad tanto en el sector de minería como de energía. Por otro lado, en Perú suele haber un ligero aumento en las publicaciones en el año 2016; sin embargo, como hay una disminución en el año 2017, no se pueda precisar una tendencia en las empresas peruanas. Esto se debe, principalmente, a que la preparación y divulgación de la información de sostenibilidad se encuentra escasamente regulada, e incluso tiende a ser voluntaria por lo general (Moneva \& Llena, 2001).

Tabla 2. Número de reportes publicados de empresas latinoamericanas de minería y energía

\begin{tabular}{|l|c|c|}
\hline \multicolumn{1}{|c|}{ País } & Número de reportes & $\%$ \\
\hline Brasil & 106 & $30 \%$ \\
\hline Colombia & 103 & $29 \%$ \\
\hline Perú & 46 & $13 \%$ \\
\hline Chile & 34 & $10 \%$ \\
\hline Argentina & 21 & $6 \%$ \\
\hline México & 17 & $5 \%$ \\
\hline Ecuador & 6 & $2 \%$ \\
\hline Uruguay & 6 & $2 \%$ \\
\hline Bolivia & 5 & $1 \%$ \\
\hline Otros & 9 & $3 \%$ \\
\hline Total & 353 & $100 \%$ \\
\hline
\end{tabular}


Figura 1. Número de publicaciones por sector y país

\begin{tabular}{|c|c|c|c|}
\hline \multirow{6}{*}{$\begin{array}{r}12 \\
10 \\
8 \\
6 \\
4 \\
2 \\
0\end{array}$} & \multicolumn{3}{|c|}{$\mathrm{N}^{\circ}$ de Reportes por país y sector } \\
\hline & \multirow{3}{*}{\multicolumn{3}{|c|}{ 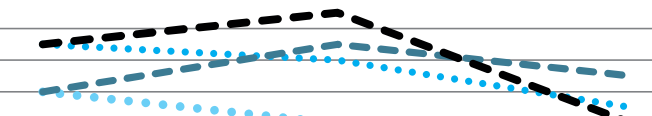 }} \\
\hline & & & \\
\hline & & & \\
\hline & & & $\boldsymbol{s}$ \\
\hline & 2015 & 2016 & 2017 \\
\hline$\ldots . .$. Chile - Energía & 6 & 4 & 2 \\
\hline ..... Chile - Minería & 9 & 8 & 5 \\
\hline - - Perú - Energía & 6 & 9 & 7 \\
\hline - - Perú - Minería & 9 & 11 & 4 \\
\hline
\end{tabular}

La mayor cantidad de publicaciones de reportes de sostenibilidad para los periodos entre 2015 y 2017 son del sector de minería. Esto se debe a que la actividad minera tiene un fuerte impacto en las comunidades campesinas y en el medioambiente. Por ello, se presenta la necesidad de rendir cuentas frente a la sociedad, las organizaciones civiles y el Estado (Slack, 2012; Jaskoski, 2014; Venegas, 2016). Sin embargo, al revisar las publicaciones de reportes de sostenibilidad para los ańos 2018 y 2019, periodos en los que era obligatorio usar los GRI Standards, se observa que solo $14 \%$ y $4 \%$ de las empresas publicaron su reporte respectivamente. Principalmente, las que más publicaron fueron las empresas peruanas del sector de energía (ver anexo 1). Por otro lado, se observa la incorporación de 23 empresas desde el periodo 2018 (ver anexo 2).

La tabla 3 muestra el nivel de cumplimiento de información medioambiental en los reportes de sostenibilidad de empresas peruanas y chilenas de acuerdo con la GRI G4. Se puede evidenciar que los aspectos en los que hay un mayor nivel de cumplimiento, considerando las escalas "informado parcialmente" e "informado totalmente", son biodiversidad, con $70 \%$ y $77 \%$ respectivamente; transporte, con $64 \%$ y $54 \%$ respectivamente; aspectos generales, con $59 \%$ y $76 \%$ respectivamente; y agua, con $57 \%$ y $60 \%$ respectivamente. Sin embargo, aparece un alto porcentaje en "no informado", lo que se debe a una falta de políticas de sostenibilidad en las empresas y la escasa influencia de entes reguladores públicos (Aranguren \& Maldonado, 2019; Del Barco et al., 2018; Rabasedas, 2018; García \& Hernández, 2019; Moneva et al., 2019; Loza, 2020).

Para el estudio cuantitativo, se realizó una regresión lineal para evaluar si los factores tamaño (número de trabajadores), cotización, exportación, sector, país y naturaleza del capital (como variables independientes) influyen significativamente en el nivel de cumplimiento de información ambiental (como variable dependiente) según este modelo. En la tabla 4, se verifica un contraste de regresión de Sig. $0.00<0,05$. Estos indicadores validan que las variables independientes en su conjunto influyen significativamente en la variable dependiente.

Los resultados de la regresión en la tabla 5 muestran qué variables tienen incidencia significativa (Sig. $<0.05)$ sobre el nivel de cumplimiento de información ambiental. Los factores tamaño de la empresa (número de trabajadores) y cotización son los que ejercen mayor influencia. 
Tabla 3. Nivel de cumplimiento de información medioambiental sobre la base de GRI G4

\begin{tabular}{|l|c|c|c|c|c|c|c|c|}
\hline \multicolumn{1}{|c|}{ Aspectos } & \multicolumn{9}{|c|}{ Perú } & \multicolumn{3}{c|}{ Chile } \\
\hline informado & $\begin{array}{c}\text { Informado } \\
\text { parcialmente }\end{array}$ & $\begin{array}{c}\text { Informado } \\
\text { totalmente }\end{array}$ & No aplica & $\begin{array}{c}\text { No } \\
\text { informado }\end{array}$ & $\begin{array}{c}\text { Informado } \\
\text { parcialmente }\end{array}$ & $\begin{array}{c}\text { Informado } \\
\text { totalmente }\end{array}$ & No aplica \\
\hline Energía & $27 \%$ & $8 \%$ & $17 \%$ & $48 \%$ & $22 \%$ & $11 \%$ & $17 \%$ & $50 \%$ \\
\hline Agua & $43 \%$ & $12 \%$ & $45 \%$ & $0 \%$ & $40 \%$ & $11 \%$ & $49 \%$ & $0 \%$ \\
\hline Emisiones & $81 \%$ & $4 \%$ & $9 \%$ & $6 \%$ & $65 \%$ & $2 \%$ & $24 \%$ & $9 \%$ \\
\hline Efluentes y residuos & $55 \%$ & $12 \%$ & $24 \%$ & $9 \%$ & $50 \%$ & $13 \%$ & $24 \%$ & $13 \%$ \\
\hline Transporte & $36 \%$ & $27 \%$ & $37 \%$ & $0 \%$ & $46 \%$ & $38 \%$ & $16 \%$ & $0 \%$ \\
\hline Reclamos & $50 \%$ & $7 \%$ & $37 \%$ & $6 \%$ & $47 \%$ & $24 \%$ & $12 \%$ & $17 \%$ \\
\hline Material & $76 \%$ & $13 \%$ & $11 \%$ & $0 \%$ & $74 \%$ & $22 \%$ & $4 \%$ & $0 \%$ \\
\hline Biodiversidad & $30 \%$ & $23 \%$ & $47 \%$ & $0 \%$ & $23 \%$ & $23 \%$ & $54 \%$ & $0 \%$ \\
\hline Cumplimiento & $19 \%$ & $9 \%$ & $24 \%$ & $48 \%$ & $8 \%$ & $15 \%$ & $24 \%$ & $53 \%$ \\
\hline Aspectos generales & $41 \%$ & $26 \%$ & $33 \%$ & $0 \%$ & $18 \%$ & $35 \%$ & $41 \%$ & $6 \%$ \\
\hline Proveedores & $77 \%$ & $17 \%$ & $6 \%$ & $0 \%$ & $80 \%$ & $17 \%$ & $3 \%$ & $0 \%$ \\
\hline
\end{tabular}

Tabla 4. Resultados de prueba ANOVA

\begin{tabular}{|c|c|c|c|c|c|c|}
\hline \multicolumn{2}{|c|}{ ANOVAa } \\
\hline \multirow{2}{*}{ Modelo } & Suma de cuadrados & Gl & Media cuadrática & F & Sig. \\
\hline & Regresión & 2,558 & 6 & 0,426 & 9,356 &, $000 \mathrm{~b}$ \\
\cline { 2 - 7 } & Residuo & 3,326 & 73 & 0,046 & & \\
\cline { 2 - 7 } & Total & 5,884 & 79 & & & \\
\hline
\end{tabular}

a. Variable dependiente: promedio final

b. Predictores: (constante) capital, exporta, país, cotiza, LN tamańo, sector

Tabla 5. Resultados de regresión lineal

\begin{tabular}{|c|c|c|c|c|c|c|}
\hline \multicolumn{7}{|c|}{ Coeficientesa } \\
\hline & \multirow{2}{*}{ Modelo } & \multicolumn{2}{|c|}{ Coeficientes no estandarizados } & \multirow{2}{*}{$\begin{array}{c}\text { Coeficientes estandarizados } \\
\text { Beta }\end{array}$} & \multirow{2}{*}{$t$} & \multirow{2}{*}{ Sig. } \\
\hline & & B & Error estándar & & & \\
\hline \multirow{7}{*}{1} & (Constante) & 0,078 & 0,218 & & 0,355 & 0,724 \\
\hline & LN Tamaño & 0,078 & 0,018 & 0,506 & 4,363 & 0,000 \\
\hline & Cotiza & $-0,129$ & 0,055 & $-0,237$ & $-2,326$ & 0,023 \\
\hline & Exporta & 0,139 & 0,082 & 0,229 & 1,685 & 0,096 \\
\hline & Sector & 0,009 & 0,080 & 0,017 & 0,116 & 0,908 \\
\hline & País & $-0,014$ & 0,053 & $-0,026$ & $-0,266$ & 0,791 \\
\hline & Capital & $-0,007$ & 0,030 & $-0,020$ & $-0,220$ & 0,827 \\
\hline
\end{tabular}

a. Variable dependiente: promedio final 
Para evaluar la significatividad de las diferencias en el nivel de cumplimiento de la información medioambiental revelada por cotización, exportación, sector, país y capital, se realizó la prueba ANOVA de un factor. La tabla 6 muestra que el sector incide significativamente $(<0.05)$ en el nivel de cumplimiento de información ambiental (promedio final). En ese caso, los factores específicos son energía, agua, emisiones, efluentes y residuos, y transporte. Es decir, las empresas mineras presentan un mayor nivel de cumplimiento de información debido al impacto que ejercen sobre las comunidades campesinas y el medioambiente. Ya que utilizan muchos recursos naturales, deben rendir cuentas sobre una actividad responsable frente a la sociedad, las organizaciones civiles y el Estado (Slack, 2012; Jaskoski, 2014).
La tabla 7 muestra que el factor exportación incide significativamente $(<0.05)$ en el nivel de cumplimiento de información ambiental (promedio final). En ese caso, los factores específicos son energía, agua, emisiones, efluentes y residuos y transporte. Es decir, las empresas que exportan presentan un mayor nivel de cumplimiento. Esto se debe a que las empresas subsidiarias de grupos transnacionales y las que realizan actividad de exportación con países desarrollados suelen tener un mayor nivel de cumplimiento con el fin de mejorar su legitimidad frente a sus socios comerciales internacionales (Perez-Batres, Miller, \& Pisani, 2010; Calixto, 2013; Alonso-Almeida et al., 2015; Hernández, 2018).

Tabla 6. Resultados ANOVA segúin sector

\begin{tabular}{|c|c|c|c|c|c|c|}
\hline \multicolumn{7}{|c|}{ ANOVA } \\
\hline & & Suma de cuadrados & gl & Media cuadrática & $\mathrm{F}$ & Sig. \\
\hline \multirow{3}{*}{ Prom energía } & Entre grupos & 3,731 & 1 & 3,731 & 24,421 & 0,000 \\
\hline & Dentro de grupos & 11,918 & 78 & 0,153 & & \\
\hline & Total & 15,649 & 79 & & & \\
\hline \multirow{3}{*}{ Prom agua } & Entre grupos & 6,582 & 1 & 6,582 & 17,371 & 0,000 \\
\hline & Dentro de grupos & 29,555 & 78 & 0,379 & & \\
\hline & Total & 36,137 & 79 & & & \\
\hline \multirow{3}{*}{ Prom emisiones } & Entre grupos & 0,918 & 1 & 0,918 & 9,715 & 0,003 \\
\hline & Dentro de grupos & 7,369 & 78 & 0,094 & & \\
\hline & Total & 8,287 & 79 & & & \\
\hline \multirow{3}{*}{$\begin{array}{l}\text { Prom efluente y } \\
\text { residuo }\end{array}$} & Entre grupos & 1,523 & 1 & 1,523 & 12,111 & 0,001 \\
\hline & Dentro de grupos & 9,812 & 78 & 0,126 & & \\
\hline & Total & 11,335 & 79 & & & \\
\hline \multirow{3}{*}{ Prom transporte } & Entre grupos & 6,147 & 1 & 6,147 & 12,302 & 0,001 \\
\hline & Dentro de grupos & 38,975 & 78 & 0,500 & & \\
\hline & Total & 45,122 & 79 & & & \\
\hline \multirow{3}{*}{ Prom final } & Entre grupos & 1,243 & 1 & 1,243 & 20,890 & 0,000 \\
\hline & Dentro de grupos & 4,641 & 78 & 0,059 & & \\
\hline & Total & 5,884 & 79 & & & \\
\hline
\end{tabular}


Tabla 7. Resultados ANOVA segúin exportación

\begin{tabular}{|c|c|c|c|c|c|c|}
\hline \multicolumn{7}{|c|}{ ANOVA } \\
\hline & & Suma de cuadrados & gl & Media cuadrática & $\mathrm{F}$ & Sig. \\
\hline \multirow{3}{*}{ Prom energía } & Entre grupos & 3,063 & 1 & 3,063 & 18,980 & 0,000 \\
\hline & Dentro de grupos & 12,586 & 78 & 0,161 & & \\
\hline & Total & 15,649 & 79 & & & \\
\hline \multirow{3}{*}{ Prom agua } & Entre grupos & 9,075 & 1 & 9,075 & 26,158 & 0,000 \\
\hline & Dentro de grupos & 27,061 & 78 & 0,347 & & \\
\hline & Total & 36,137 & 79 & & & \\
\hline \multirow{3}{*}{ Prom emisiones } & Entre grupos & 0,813 & 1 & 0,813 & 8,483 & 0,005 \\
\hline & Dentro de grupos & 7,474 & 78 & 0,096 & & \\
\hline & Total & 8,287 & 79 & & & \\
\hline \multirow{3}{*}{$\begin{array}{l}\text { Prom efluente y } \\
\text { residuo }\end{array}$} & Entre grupos & 1,372 & 1 & 1,372 & 10,743 & 0,002 \\
\hline & Dentro de grupos & 9,963 & 78 & 0,128 & & \\
\hline & Total & 11,335 & 79 & & & \\
\hline \multirow{3}{*}{ Prom transporte } & Entre grupos & 6,129 & 1 & 6,129 & 12,261 & 0,001 \\
\hline & Dentro de grupos & 38,993 & 78 & 0,500 & & \\
\hline & Total & 45,122 & 79 & & & \\
\hline \multirow{3}{*}{ Prom final } & Entre grupos & 1,272 & 1 & 1,272 & 21,508 & 0,000 \\
\hline & Dentro de grupos & 4,612 & 78 & 0,059 & & \\
\hline & Total & 5,884 & 79 & & & \\
\hline
\end{tabular}

La tabla 8 muestra que el país no incide significativamente $(>0.05)$ en el nivel de cumplimiento de información ambiental (promedio final). Sin embargo, al analizar los factores de forma individual, el aspecto emisiones sí presenta una incidencia en el nivel de cumplimiento; concretamente, las empresas chilenas tienen mayor cumplimiento. El marco regulador de los aspectos medioambientales en Chile es establecido y controlado principalmente por el Ministerio del Medio Ambiente, cuya función es el diseño y aplicación de políticas, planes y programas en materia ambiental; asimismo, comprende la protección y con- servación de la diversidad biológica, y de los recursos naturales renovables e hídricos.

La tabla 9 muestra que el factor cotización no incide significativamente $(>0.05)$ en el nivel de cumplimiento de información ambiental (promedio final). Sin embargo, al analizar los factores de forma individual, solo los aspectos emisiones y reclamos sí presentan una incidencia. Finalmente, el factor naturaleza del capital tampoco muestra una incidencia en el nivel de cumplimiento de información ambiental (promedio final) ni en los aspectos de forma individual. 
Tabla 8. Resultados ANOVA según país

\begin{tabular}{|l|l|c|c|c|c|c|}
\hline \multicolumn{9}{|c|}{ ANOVA } \\
\hline \multirow{3}{*}{ Prom emisiones } & Suma de cuadrados & gl & Media cuadrática & F & Sig. \\
\cline { 2 - 7 } & Entre grupos & 1,755 & 1 & 1,755 & 20,954 & $\mathbf{0 , 0 0 0}$ \\
\cline { 2 - 7 } & Dentro de grupos & 6,532 & 78 & 0,084 & & \\
\cline { 2 - 7 } & Total & 8,287 & 79 & & 3,416 & $\mathbf{0 , 0 6 8}$ \\
\hline \multirow{3}{*}{ Prom final } & Entre grupos & 0,247 & 78 & 0,247 & \\
\cline { 2 - 7 } & Dentro de grupos & 5,637 & 79 & & & \\
\cline { 2 - 7 } & Total & 5,884 & & & \\
\end{tabular}

Tabla 9. Resultados ANOVA según cotización

\begin{tabular}{|c|c|c|c|c|c|c|}
\hline \multicolumn{7}{|c|}{ ANOVA } \\
\hline & & Suma de cuadrados & gl & Media cuadrática & $\mathrm{F}$ & Sig. \\
\hline \multirow{3}{*}{ Prom emisiones } & Entre grupos & 1,102 & 1 & 1,102 & 11,967 & 0,001 \\
\hline & Dentro de grupos & 7,184 & 78 & 0,092 & & \\
\hline & Total & 8,287 & 79 & & & \\
\hline \multirow{3}{*}{ Prom reclamos } & Entre grupos & 4,967 & 1 & 4,967 & 6,791 & 0,011 \\
\hline & Dentro de grupos & 50,470 & 69 & 0,731 & & \\
\hline & Total & 55,437 & 70 & & & \\
\hline \multirow{3}{*}{ Prom final } & Entre grupos & 0,200 & 1 & 0,200 & 2,750 & 0,101 \\
\hline & Dentro de grupos & 5,684 & 78 & 0,073 & & \\
\hline & Total & 5,884 & 79 & & & \\
\hline
\end{tabular}

Los resultados pueden ser sustentados por la teoría de la legitimidad, ya que $96 \%$ de las memorias revisadas revelan información referente a mejoras en las prácticas y desempeño ambiental, logros destacados, premios recibidos referentes a sostenibilidad, disminuciones en emisiones de CO2 o del GEI, disminuciones de consumo de agua y de electricidad, etc. Las empresas difunden información sobre sus mejoras para cambiar la imagen y la percepción que las comunidades tienen sobre ellas, con el objetivo de aumentar la legitimidad de su accionar (Cho \& Patten, 2007; Kuruppu \& Milne, 2016; Pajares et al., 2016; Venegas, 2016; Asmeri et al., 2017). Además, la divulgación de información medioambiental dependerá de la presión social y política que ejerzan las comunidades con respecto a su desempeño (Cho \& Patten, 2007; Kuruppu \& Milne, 2016; Venegas, 2016; Asmeri et al., 2017). La tabla 10 muestra los aspectos referentes a legitimidad más revelados por las empresas mineras y de energía. 
Tabla 10. Resultados de legitimidad

\begin{tabular}{|c|c|}
\hline Aspectos & Porcentaje \\
\hline $\begin{array}{l}\text { Revegetación de hectáreas, realización de plantones y programas de reforestación, cuidado y reservación de fauna, reubi- } \\
\text { cación de especies, etc. }\end{array}$ & $84 \%$ \\
\hline $\begin{array}{l}\text { Gestión eficiente de residuos por medio de reutilización, compostaje, reciclaje, recuperación, almacenamiento, etc. (reali- } \\
\text { zado por la misma empresa o a través de terceros) }\end{array}$ & $71 \%$ \\
\hline $\begin{array}{l}\text { Inversión en mejoras de protección ambiental, como en tratamiento de residuos, reducción de emisiones, y prevención y } \\
\text { gestión ambiental }\end{array}$ & $66 \%$ \\
\hline $\begin{array}{l}\text { Evaluación del cumplimiento de los proveedores con los criterios ambientales y de su desempeño, y búsqueda de mejora } \\
\text { continua }\end{array}$ & $65 \%$ \\
\hline $\begin{array}{l}\text { Gestión responsable del agua a través de la reutilización y/o recirculación de esta, así como monitoreos de la cantidad y } \\
\text { calidad de agua usada }\end{array}$ & $63 \%$ \\
\hline $\begin{array}{l}\text { Diversas mejoras a fin de minimizar los impactos ambientales generados, como programas de innovación tecnológica para } \\
\text { reducción de impactos ambientales, ideas creativas de colaboradores, mejoras en procesos, etc. }\end{array}$ & $57 \%$ \\
\hline Mitigación de impactos ambientales del transporte de recursos, trabajadores, etc. & $53 \%$ \\
\hline $\begin{array}{l}\text { Diferentes logros como la renovación de las certificaciones internacionales ISO } 9001 \text { (Gestión de la Calidad), ISO } 14001 \\
\text { (Gestión Ambiental) y OHSAS } 18001 \text { (Gestión de Seguridad y Salud en el Trabajo), o mantenerse en el registro de OEFA }\end{array}$ & $45 \%$ \\
\hline Indicación del número de reclamos ambientales atendidos y las medidas de mejora realizadas & $41 \%$ \\
\hline Reducción del consumo de energía al optar por una energía renovable, por lo que se reduce la contaminación & $38 \%$ \\
\hline $\begin{array}{l}\text { Recepción de premios y distintivos, como "Empresa Socialmente Responsable", premio "Las } 5 \text { S", "Yo reciclo", "Premio } \\
\text { Nacional de Minería", "Excelencia en Prevención de Riesgos", "Top Green Companies", etc. }\end{array}$ & $30 \%$ \\
\hline $\begin{array}{l}\text { Compromiso con una gestión de seguridad por medio de la búsqueda de medidas correctivas, como capacitaciones y } \\
\text { aumento de controles para lograr la reducción de los índices de accidentalidad en el personal con el fin de lograr cero } \\
\text { daños }\end{array}$ & $30 \%$ \\
\hline $\begin{array}{l}\text { Gestión para la reducción de la huella del carbono, las emisiones del efecto invernadero, diferentes gases contaminantes y } \\
\text { la contaminación sonora, por medio del monitoreo de los límites máximos permisibles }\end{array}$ & $28 \%$ \\
\hline
\end{tabular}

\section{Conclusiones}

Perú y Chile ocupan el tercer y cuarto lugar respectivamente en cuanto a la cantidad de reportes de sostenibilidad publicados en la base de datos de la GRI en los periodos entre 2015 y 2017 en los sectores de minería y energía. Sin embargo, dicha práctica no ha sido uniforme, pues la frecuencia de publicación de las empresas no ha sido periódica.

Se puede evidenciar que los aspectos en los que hay un mayor nivel de cumplimiento de acuerdo con la GRI G4, considerando las ponderaciones "informado parcialmente" e "informado totalmente", son biodiversidad, transporte, aspectos generales y agua. Sin embargo, existe un alto porcentaje de "no informado" en todos los aspectos ambientales, lo que se debe a una falta de políticas de sostenibilidad en las empresas y a la escasa influencia de entes reguladores públicos (Aranguren \& Maldonado, 2019; Del Barco et al., 2018; Rabasedas, 2018; García \& Hernández, 2019; Moneva et al., 2019; Loza, 2020). Para los periodos posteriores a los analizados en la presente investigación, se observa que un porcentaje reducido de las empresas continuaron publicando sus reportes de sostenibilidad en la base 
de datos de la GRI de acuerdo con los GRI-Standards: $14 \%$ en el año 2018 y $4 \%$ en 2019.

Es importante analizar la sostenibilidad corporativa en la industria extractiva (minería, gas, petróleo y energía), ya que es la que presenta los mayores problemas ambientales y sociales que cubren la mayoría de los indicadores de sostenibilidad. Además, en el caso de las empresas mineras, la influencia de las comunidades campesinas y sus reclamos obligan a la divulgación de sostenibilidad ambiental para rendir cuentas y justificar su desempeño (Slack, 2012; Jaskoski, 2014).

El 96\% de los reportes de sostenibilidad analizados divulga información positiva de desempeño ambiental. Destacan las actividades realizadas para cuidar la biodiversidad, las mejoras en la gestión responsable del agua y residuos, etc. También resaltan las inversiones realizadas. Esto se debe principalmente a la búsqueda de las empresas por ratificarse y mejorar su reputación, sobre todo, cuando perciben que su legitimidad se ve amenazada por las preocupaciones del público con respecto a las repercusiones de su actividad en el entorno (Ahmad \& Sulaiman, 2004; Branco et al., 2008; Cho, 2009; Venegas, 2016; Dube \& Maroun, 2017).

Las variables independientes cotización y tamaño (medido por número de trabajadores) son factores determinantes que ejercen una influencia en el nivel de cumplimiento de información ambiental. El nivel de cumplimiento resulta mayor en las grandes empresas que poseen más recursos, y realizan mayores inversiones en temas ambientales y mejoras porque tienden a tener desarrolladas sus estrategias corporativas de sostenibilidad en la sociedad (Hernández, 2016). La variable cotización resulta significativa, pues las empresas cotizadas tendrán un mayor número de usuarios de sus estados financieros que exigirán más información usualmente distinta a la obligatoria (Ponce \& Labat, 2011).
Las implicancias de este estudio refieren una necesidad de analizar más sectores representativos, así como otros factores. Entre ellos, se encuentran el enfoque estratégico, la rentabilidad, la actitud de gerentes o la aplicación de otra teoría de divulgación de información de sostenibilidad.

\section{Referencias bibliográficas}

Ahmad, N. N., \& Sulaiman, M. (2004). Environmental Disclosures in Malaysian Annual Reports: A Legitimacy Theory Perspective. International Journal of Commerce \& Management, 24(1), 44-58. https://doi.org/ $10.1108 / 10569210480000173$

Alonso-Almeida, M., Marimon, F., \& Llach, J. (2015). Difusión de las memorias de sostenibilidad en Latinoamérica: análisis territorial y sectorial. Estudios Gerenciales, 31(135), 139-149. https://doi.org/10.1016/j. estger.2015.01.002

Alzate, Y. M. S., Zapata, G. M. V., \& Gómez, M. J. B. (2018). Un análisis de los estudios acerca de las memorias de sostenibilidad en Latinoamérica. Contabilidad y Negocios, 13(26), 56-73. https://doi.org/10.18800/ contabilidad.201802.004

Aranguren, N., \& Maldonado, S. (2019). Governance and Type of Industry as Determinants of Corporate Social Responsibility Disclosures in Latin America. Latin American Business Review, 21(1), 1-35. https://doi.org /10.1080/10978526.2019.1697185

Aras, G., \& Crowther, D. (2009). Corporate Sustainability Reporting: A Study in Disingenuity? Journal of Business Ethics, 87, 279-288. https://doi.org/10.1007/ s10551-008-9806-0

Archel, P., \& Lizarraga, F. (2001). Algunos determinantes de la información medioambiental divulgada por las empresas espańolas cotizada. Revista de Contabilidad, 4(7), 139-153.

Archel, P. (2003): La divulgación de la información social y medioambiental de la gran empresa española en

Contabilidad y Negocios (16) 31, 2021 / e-ISSN 2221-724X 
el período 1994-1998: situación actual y perspectivas. Revista Española de Financiación y Contabilidad, 32(117), 571-601. https://doi.org/10.1080/0210241 2.2003.10779496

Asmeri, R., Alvionita, T., \& Gunardi, A. (2017). CSR Disclosures in the Mining Industry: Empirical Evidence from Listed Mining Firms in Indonesia. Indonesian Journal of Sustainability Accounting and Management, 1(1), 16-22. https://doi.org/10.28992/ijsam.v1i1.23

Asociación Española de Contabilidad y Administración de Empresas. (AECA) (2004). Marco conceptual de la responsabilidad social corporativa. Recuperado de https:// www.aeca.es/old/pub/documentos/rs1.htm [Consulta: 25 de abril de 2019].

Asociación Española para la Calidad (AEC). (2008). Sistemas de gestión de la calidad [Norma UNE-EN ISO 9001].

Bebbington, A., \& Bury, J. (2009). Institutional Challenges for Mining and Sustainability in Peru. Proceedings of the National Academy of Sciences, 106(41), 1729617301. https://doi.org/10.1073/pnas.0906057106

Bengua, J., \& Clerici, Y. (2009). Responsabilidad social empresarial: análisis de las memorias en Argentina. Gestión Joven, 72(4), 1-21.

Böhling, K., Murguia, D., \& Godfried, J. (2017). Sustainability Reporting in the Mining Sector: Exploring its Symbolic Nature. Business and Society, 58(1), 191225. https://doi.org/10.1177/0007650317703658

Boiral, O., \& Henri, J. F. (2017). Is Sustainability Performance Comparable? A Study of GRI Reports of Mining Organizations. Business \& Society, 56(2), 283317. https://doi.org/10.1177/0007650315576134

Branco, M., Eugénio, T., Ribeiro, J., Brammer, S. J., \& Pavelin, S. (2008). Environmental Disclosure in Response to Public Perception of Environmental Threats: The Case of Co-Incineration in Portugal. Journal of Communication Management, 12(2), 136-151. https:// doi.org/10.1108/13632540810881956
Brown, N., \& Deegan, C. (1998). The Public Disclosure of Environmental Performance Information - A Dual Test of Media Agenda Setting Theory and Legitimacy Theory. Accounting and Business Research, 29(1), 21-41. https://doi.org/10.1080/00014788.1998.9729564

Buchanan, S., \& Marques, J. C. (2018). How Home Country Industry Associations Influence MNE International CSR Practices: Evidence from the Canadian Mining Industry. Journal of World Business, 53(1), 63-74. https://doi.org/10.1016/j.jwb.2017.07.005

Calixto, L. (2013). A divulgação de relatórios de sustentabilidade na América Latina: um estudo comparativo. Revista de Administração, 48(4), 828-842. https://doi. org/10.5700/rausp1124

Cho, C. H. (2009). Legitimation Strategies Used in Response to Environmental Disaster: A French Case Study Total SA's Erika and AZF Incidents. European Accounting Review, 18(1), 33-62. https://doi.org/10.1080/ 09638180802579616

Cho, C. H., \& Patten, D. M. (2007). The Role Environmental Disclosures as Tools of Legitimacy: A Research Note. Accounting, Organizations and Society, 32(7), 639-647. https://doi.org/10.1016/j.aos.2006.09.009

Clarckson, P., Li, Y., Richardson, G., \& Vasvari, F. (2008). Revisiting the Relation between Environmental Performance and Environmental Disclosure: An Empirical Analysis. Accounting, Organizations and Society, 33(4-5), 303-327. https://doi.org/10.1016/j.aos.2007.05.003

Cooke, T. (1989). Voluntary Corporate Disclosure by Swedish Companies. Journal of International Financial Management \& Accounting, 1(2), 171-195. https:// doi.org/10.1111/j.1467-646X.1989.tb00009.x

Cormier, D., \& Gordon, I. M. (2001). An Examination of Social and Environmental Reporting Strategies. Accounting, Auditing and Accountability Journal, 14(5), 587616. https://doi.org/10.1108/EUM0000000006264

Cormier, D., \& Magnan, M. (2003). Environmental Reporting Management: A Continental European Perspective. 
Journal of Accounting and Public Policy, 22(1), 43-62. https://doi.org/10.1016/S0278-4254(02)00085-6

Corrigan, C. C. (2018). Corporate Social Responsibility and Local Context: The Case of Mining in Southern Africa. Resources Policy, 55(C), 233-243. https://doi. org/10.1016/j.resourpol.2017.12.007

Da Silva, S., \& Aibar, B. (2011). Factores determinantes del grado de información medioambiental divulgada en las grandes empresas que operan en Portugal: un análisis univariante. Base Revista de Administração e Contabilidade da UNISINOS, 8(1), 3-19. https://doi. org/10.4013/base.2011.81.01

Dashwood, H. S. (2012). The Rise of Global Corporate Social Responsibility. Mining and the Spread of Global Norms. Cambridge: Cambridge University Press. https://doi. org/10.1017/CBO9781139058933

Daub, C. H. (2007). Assessing the Quality of Sustainability Reporting: An Alternative Methodological Approach. Journal of Cleaner Production, 15(1), 75-85. https:// doi.org/10.1016/j.jclepro.2005.08.013

De Waal, A. A., \& Orcotoma, G. (2011). Does the Application of Corporate Social Responsibility Support a HighPerformance Organization in Achieving Better Results? The case of Mining Multinationals in Peru. International Journal of Sustainable Strategic Management, 3(1), 33-49. https://doi.org/10.1504/IJSSM.2011.040778

Del Barco, J. P., Del Barco, M. A., Del Barco, M. S., \& Federici, I. (2018). Divulgación de aspectos ambientales en los informes de sostenibilidad frente al nuevo escenario internacional. CAPIC REVIEW, 16(1), 1-19. https://doi.org/10.35928/cr.vol16.2018.67

Deegan, C. (2002). The Legitimising Effect of Social and Environmental Disclosures. A Theoretical Foundation. Accounting, Auditing and Accountability Journal, 15(3), 282-311. https://doi.org/10.1108/09513570 210435852

Deegan, C., \& Gordon, B. (1996). A Study of the Environmental Disclosures Practices of Australian Corporations.
Accounting and Accountability Journal, 26(3), 36-51. https://doi.org/10.1080/00014788.1996.9729510

Deegan, C., Rankin, M., \& Tobin, J. (2002). An Examination of the Corporate Social and Environmental Disclosures of BHP from 1983-1997. A test of Legitimacy Theory. Accounting, Auditing and Accountability Journal, 15(3), 312-343. https://doi. org/10.1108/09513570210435861

Djakman, C. D, \& Machmud, N. (2008, 23-24 de julio). Pengaruh Struktur Kepemilikan terhadapLuas Pengungkapan Tanggung Jawab Sosial (CSR Disclosure) pada Laporan TahunanPerusahaan: Studi Empiris pada PerusahaanPublik yang Tercatat di Bursa Efek IndonesiaTahun 2006 [Presentación en simposio]. Simposium Nasional Akuntansi XI, Pontianak, Indonesia.

Dong, S., \& Xu, L. (2016). The Impact of Explicit CSR Regulation: Evidence from China's Mining Firms. Journal of Applied Accounting Research, 17(2), 237258. https://doi.org/10.1108/JAAR-03-2014-0030

Dube, S., \& Maroun, W. (2017). Corporate Social Responsibility Reporting by South African Mining Companies: Evidence of Legitimacy Theory. South African Journal of Business Management, 48(1), 23-34. https:// doi.org/10.4102/sajbm.v48i1.17

Fonseca, A., McAllister, M. L., \& Fitzpatrick, P. (2014). Sustainability Reporting among Mining Corporations: A Constructive Critique of the GRI Approach. Journal of Cleaner Production, 84(1), 70-83. https:// doi.org/10.1016/j.jclepro.2012.11.050

Freedman, M., \& Jaggi, B. (2005). Global Warming, Commitment to Kyoto Protocol, and Accounting Disclosures by the Largest Global Public Firms from Polluting Industries. The International Journal of Accounting, 40(3), 215-232. https://doi.org/10.1016/j. intacc.2005.06.004

García, C., \& Hernández, J. (2019, 11-13 de setiembre). Factores de información de sostenibilidad en páginas web y reporte obligatorio para empresas cotizadas peruanas [Presentación en conferencia]. 12th Spanish

Contabilidad y Negocios (16) 31, 2021 / e-ISSN 2221-724X 
Conference on Social and Environmental Accounting Research, Pamplona, España.

Gifford, B., \& Kestler, A. (2008). Toward a Theory of Local Legitimacy by MNEs in Developing Nations: Newmont Mining and Health Sustainable Development in Peru. Journal of International Management, 14(4), 340352. https://doi.org/10.1016/j.intman.2007.09.005

Global Reporting Initiative (GRI). (2016). Guía para la elaboración de memorias de sostenibilidad. Recuperado de http://www.mas-business.com/docs/Spanish-G4.pdf [Consulta: 3 de mayo de 2019].

Gray, R., Kouhy, R., \& Lavers, S. (1995a). Corporate Social and Environmental Reporting. A Review of the Literature and a Longitudinal Study of UK Disclosure. Accounting, Auditing and Accountability Journal, 8(2), 47-77. https://doi.org/10.1108/09513579510146996

Gray R., Kouhy, R., \& Lavers, S. (1995b). Methodological Themes: Constructing a Research Database of Social and Environmental Reporting by UK Companies. Accounting, Auditing \& Accountability, 8(2), 78-101. https://doi.org/10.1108/09513579510086812

Gray, R., Owen, D. L., \& Adams, C. A. (1996). Accounting and Accountability: Changes and Challenges in Corporate Social and Environmental Reporting. Londres: Prentice Hall.

Guenther, E., Hoppe, H., \& Poser, C. (2006). Environmental Corporate Social Responsibility of Firms in the Mining and Oil and Gas Industries: Current Status Quo of Reporting Following GRI Guidelines. Greener Management International, (53), 7-25. https:// doi.org/10.9774/GLEAF.3062.2006.sp.00003

Hernández, J. (2008, 27-28 de agosto). Información de sostenibilidad en los informes anuales de las empresas mineras en el Perú [Presentación en foro]. Foro Virtual de Contabilidad Ambiental y Social, Buenos Aires, Argentina.

Hernández, J. (2016). Estado de la situación de la información de sostenibilidad de las empresas peruanas. Revista Contabilidad \& Sistemas, 9, 47-55. https://doi. org/10.35928/cr.vol15.2017.5
Hernández, J. (2018). Influencia de la naturaleza internacional de empresas peruanas en su información de sostenibilidad. Revista de Comunicación, 17(1), 74-92. https://doi.org/10.26441/RC17.1-2018-A4

Husillos, F. J. (2007). Una aproximación desde la teoría de la legitimación a la información medioambiental revelada por las empresas españolas cotizadas. Revista Española de Financiación y Contabilidad, 37(133), 97-122. https://doi.org/10.1080/02102412.2007.10779616

Islam, M. A., \& Deegan, C. (2010). Media Pressures and Corporate Disclosure of Social Responsibility Performance Information: A study of Two Global Clothing and Sports Retail Companies. Accounting and Business Research, 40(2), 131-148. https://doi.org/10.1080/00 014788.2010.9663388

Jaskoski, M. (2014). Environmental Licensing and Conflict in Peru's Mining Sector: A Path-Dependent Analysis. World Development, 64, 873-883. https:// doi.org/10.1016/j.worlddev.2014.07.010

Jenkins, H. (2004). Corporate Social Responsibility and the Mining Industry: Conflicts and Constructs. Corporate Social Responsibility and Environmental Management, 11(1), 23-34. https://doi.org/10.1002/csr.50

Jenkins, H., \& Yakovleva, N. (2006). Corporate Social Responsibility in the Mining Industry: Exploring Trends in Social and Environmental Disclosure. Journal of Cleaner Production, 14(3-4), 271-284. https://doi. org/10.1016/j.jclepro.2004.10.004

KPMG. (2011). KPMG International Survey of Corporate Responsibility Reporting 2011. Recuperado de https:// assets.kpmg/content/dam/kpmg/pdf/2012/02/Corporate-responsiblity-reporting-2012-eng.pdf [Consulta: 5 de mayo de 2019].

Kuruppu, S., \& Milne, M. J. (2016, 13-15 de julio). When to Report and When not to Report: Understanding How a Company Manages Reputation to Maintain Legitimacy [Presentación en conferencia]. 8th Asia Pacific Interdisciplinary Research in Accounting Conference, Melbourne, Australia. 
Leuz, C. (2000). The Development of voluntary Cash Flow Statements in Germany and the Influence of International Reporting Standards. Schmalebach Business Review, 52, 182-207. https://doi.org/10.1007/BF03396616

Lodhia, S., \& Hess, N. (2014). Sustainability Accounting and Reporting in the Mining Industry: Current Literature and Directions for Future Research. Journal of Cleaner Production, 84, 43-50. https://doi. org/10.1016/j.jclepro.2014.08.094

Loza, C. R. (2020). Sustainability Reporting Quality of Peruvian Listed Companies and the Impact of Regulatory Requirements of Sustainability Disclosures. Sustainability, 12(3), 1-22. https://doi.org/10.3390/su12031135

Marimon, F., Alonso-Almeida, M., Rodríguez, M., \& Alejandro, K. (2012). The Worldwide Diffusion of the Global Reporting Initiative: What is the Point? Journal of Cleaner Production, 33, 132-144. https://doi. org/10.1016/j.jclepro.2012.04.017

Mathews, M. R. (1997): Twenty-Five Years of Social and Environmental Accounting Research: Is There a Silver Jubilee to Celebrate? Accounting, Auditing and Accountability Journal, 10(4), 481-531. https://doi. org/10.1108/EUM0000000004417

Meek, G., Roberts, C., \& Gray, S. (1995). Factors Influencing Voluntary Annual Report Disclosures by US, UK and Continental European Multinational Corporations. Journal of International Business, 26(3), 555572. https://doi.org/10.1057/palgrave.jibs.8490186

Ministerio de Economía y Minas (MEM). (2017) Anuario Minero Perú 2017. Recuperado de https://www.gob. pe/minem [Consulta: 15 de abril de 2019].

Momin, M. A., \& Parker, L. D. (2013). Motivations for Corporate Social Responsibility Reporting by MNC Subsidiaries in an Emerging Country: The Case of Bangladesh. The British Accounting Review, 45(3), 215-228. https://doi.org/10.1016/j.bar.2013.06.007

Moneva J.M., Acero I., \& Llena F. (2007) Evaluación de la información de sostenibilidad en las cajas de ahorro españolas. Cuadernos Aragoneses de Economía, 17(1), 99-125.

Moneva, J. M, Archel, P., \& Correa, C. (2006). GRI and the Camouflaging of Corporate Unsustainability. Accounting Forum, 30(2), 121-137. https://doi.org/ 10.1016/j.accfor.2006.02.001

Moneva, J.M., Jara-Sarrúa, L., Hernández, J., \& Del Barco, J. P. (2019). The Disclosure of Social Issues in Latin American Sustainability Reports: An Exploration of their Disclosure in Argentina, Chile, and Peru. En I. M. García-Sánchez \& J. Martínez-Ferrero (Eds.), The Disclosure and Assurance of Corporate Social Responsibility: A Growing Market (pp.135-174). Cambridge: Cambridge Scholars Publishing.

Moneva, J.M., \& Llena, F. (2001). Environmental Disclosures in the Annual Reports of Larges Companies in Spain. The European Accounting Review, 9(1), 7-29. https://doi.org/10.1080/096381800407923

Nakasone, G. (2015). Contabilidad ambiental: una propuesta basada en los reportes de sostenibilidad en las industrias minera, petrolera y de gas. Contabilidad y Negocios, 10(19), 5-26.

Navarro, V., \& Denis, C. (2003, 5-7 de noviembre). Environmental, Health and Safety Management Systems for Underground Mining [Presentación en conferencia]. 1st International Conference on Sustainable Development and Management of Surface, Utrecht, Países Bajos.

O'Donovan, G. (2002). Environmental Disclosures in the Annual Report. Extending the Applicably and Predictive Power of Legitimacy Theory. Accounting, Auditing and Accountability Journal, 15(3), 344-370. https:// doi.org/10.1108/09513570210435870

Pajares, J. Morales, C. V., \& Pintado, L. A. (2016). Factores de información medioambiental de las empresas mineras peruanas. CAPIC REVIEW, 14(1), 29-38. https://doi.org/10.35928/cr.vol14.2016.30

Park, B. I., \& Ghauri, P. N. (2015). Determinants Influencing CSR Practices in Small and Medium Sized 
MNE Subsidiaries: A Stakeholder Perspective. Journal of World Business, 50(1), 192-204. https://doi. org/10.1016/j.jwb.2014.04.007

Peck, P., \& Sinding, K. (2003). Environmental and Social Disclosure and Data Richness in the Mining Industry. Business Strategy and the Environment, 12(3), 131-146. https://doi.org/10.1002/bse.358

Perez, F., \& Sanchez, L.E. (2009). Assessing the Evolution of Sustainability Reporting in the Mining Sector. Environmental Management, 43(6), 949-961. https:// doi.org/10.1007/s00267-008-9269-1

Perez-Batres, L., Miller, V., \& Pisani, M. (2010). CSR, Sustainability and the Meaning of Global Reporting for Latin American Corporations. Journal of Business Ethics, 91(2), 193-209. https://doi.org/10.1007/ s10551-010-0614-y

Ponce, H. G., \& Labat, B. N. (2011). La revelación de información social por parte de las empresas españolas: factores explicativos y necesidad de legitimidad social. Contabilidad y Negocios, 6(12), 5-25.

Porro, M. (2008). Corporate Social Responsibility in Peru (Responsabilidad social empresarial en el Perú). Revist@e-Mercatoria, 7(1), 1-14.

Purwanto, A. (2011). Pengaruh Tipe Industri Ukuran Perusahaan, Profitabilitas terhadap Corporate Social Responsibility. Jurnal Akuntansi \& Auditing, 8(1),1-94.

Quiroz, P. (2008). Responsabilidad social empresarial como base para el desarrollo sostenible en el Perú. Gestión Joven, 3, 89-96

Rabasedas, M. (2018). Divulgación de información medioambiental: análisis exploratorio para las entidades cotizadas en Argentina. CAPIC REVIEW, 16, 1-16. https://doi.org/10.35928/cr.vol16.2018.63

Riantani, S., \& Nurzamzam, H. (2015). Analysis of Company Size, Financial Leverage, and Profitability and It's Effect to CSR Disclosure. Jurnal Dinamika Manajemen, 6 (2), 203-213. https://doi.org/10.15294/jdm. v6i2.4308
Saleh, M., Zulkifli, N \& Muhamad, R. (2010). Corporate Social Responsibility Disclosure and Its Relation on Institutional Ownership. Managerial Auditing Journal, 25(6), 591-613. https://doi.org/10.1108/02686 901011054881

Sembiring, E. R. (2005, 15-16 de setiembre). Karakteristik Perusahaan dan Pengungkapan Tanggung Jawab Sosial: Study Empiris pada Perusahaan yang Tercatat di Bursa Efek Jakarta [Presentación en simposio]. Simposium Nasional Akuntansi VIII Solo, Yakarta, Indonesia.

Servicio Nacional de Geología y Minería (SERNAGEOMIN). (2017). Anuario Minero Chile 2017. Recuperado de https://www.sernageomin.cl/ [Consulta: 15 de abril de 2019].

Slack, K. (2012). Mission impossible? Adopting A CSRBased Business Model for Extractive Industries in Developing Countries. Resources Policy, 37(2), 179-184. https://doi.org/10.1016/j.resourpol.2011.02.003

Szczepankiewicz, E., \& Mućko, P. (2016). CSR Reporting Practices of Polish Energy and Mining Companies. Sustainability, 8(2), 1-17. https://doi.org/10.3390/su8020126

Sweeney, L., \& Coughlan, J. (2008). Do Different Industries Report Corporate Social Responsibility Differently? An Investigation through the Lens of Stakeholder Theory. Journal of Marketing Communications, 14(2), 113-124. https://doi.org/10.1080/13527260701856657

Tost, M., Hitch, M., Chandurkar, V., Moser, P., \& Feiel, S. (2018). The State of Environmental Sustainability Considerations in Mining. Journal of Cleaner Production, 182, 969-977. https://doi.org/10.1016/j.jclepro.2018.02.051

Tostes, M., \& Chero, L. C. (2010). Análisis comparativo de la responsabilidad social en el sector financiero: Estudios de caso en Perú y Brasil 2007-2009. Derecho PUCP, (64), 299-315. https://doi.org/10.18800/ derechopucp.201001.017

Unerman, J., Bebington, J., \& O’Dwyer, B. (2007). Sustainability, Accounting and Accountability. Nueva York: Routledge. 
Van Staden, C., \& Hooks, J. (2007). A Comprehensive Comparison of Corporate Environmental Reporting and Responsiveness. The British Accounting Review, 39(3), 197-210. https://doi.org/10.1016/j.bar.2007. 05.004

Venegas, M. C. (2016). Factores y características de la información de desempeño ambiental en los estados financieros e informes de sostenibilidad en las empresas mineras peruanas (Tesis de doctorado, Facultad de Ciencias Económicas y Estadística, Universidad Nacional de Rosario. Rosario, Argentina).

Vintró, C., \& Comajuncosa, J. (2009). La responsabilidad social corporativa en la minería. En Cámara Oficial Mineira Galicia (coord.), Minería sostenible: conferencia internacional (pp. 835-849). La Coruña: Cámara Oficial Mineira Galicia

Viveros, H. (2016). Examining Stakeholders' Perceptions of Mining Impacts and Corporate Social Responsibility. Corporate Social Responsibility and Environmental Management, 23(1), 50-64. https://doi.org/10.1002/ csr. 1363
Warhurst, A. (2001). La ciudadanía corporativa y de inversión social de las empresas: los conductores de alianzas trisectoriales. Journal of Corporate Citizenship, 1, 57-73. https://doi.org/10.9774/GLEAF.4700.2001.sp.00008

Warsame, H., Simmons, C. V., \& Neu, D. (2002). Responding to 'discrediting' events: Annual report disclosure responses to environmental fines. Accounting and the Public Interest, 2(1), 22-40. https://doi.org/10.2308/ api.2002.2.1.22

Williams, J. (1999). Green and Social Issues and the Management Accountant. Management Accounting, 1, 64-65.

Wilmshurst, T. D., \& Frost, G. R. (2000). Corporate Environmental Reporting. A Test of Legitimacy Theory. Accounting, Auditing and Accountability Journal, 13(1), 10-26.https://doi.org/10.1108/09513570010316126

Wirth, H., Kulczycka, J., Hausner, J., \& Koński, M. (2016). Corporate Social Responsibility: Communication about Social and Environmental Disclosure by Large and Small Copper Mining Companies. Resources Policy, 49, 53-60. https://doi.org/10.1016/j.resourpol.2016.04.007

\section{ANEXOS}

Anexo 1. Empresas que continuaron divulgando en GRI database con GRI Standards

\begin{tabular}{|l|l|l|l|l|}
\hline \multicolumn{1}{|c|}{ Empresa } & \multicolumn{1}{c|}{ País } & \multicolumn{1}{c|}{ Sector } & \multicolumn{1}{c|}{2018} \\
\hline Milpo & Perú & Minería & GRI Standards & 0 \\
\hline Yanacocha & Perú & Minería & Citing GRI & 0 \\
\hline Codelco & Chile & Minería & GRI Standards & 0 \\
\hline CELEPSA Perú & Perú & Energía & GRI Standards & GRI Standards \\
\hline CENERGIA & Perú & Energía & GRI Standards & \\
\hline Contugas & Perú & Energía & GRI Standards & GRI Standards \\
\hline Electroperú & Perú & Energía & GRI Standards & 0 \\
\hline Electro Sur Este & Perú & Energía & GRI Standards & 0 \\
\hline Enel Generación Piura & Perú & Energía & GRI STANDARDS & 0 \\
\hline Repsol Perú & Perú & Energía & GRI STANDARDS & \\
\hline SATEL PERU & Perú & Energía & GRI STANDARDS & GRI Standards \\
\hline
\end{tabular}

Fuente: Elaboración propia. 
Anexo 2. Empresas que empezaron a divulgar en GRI database para periodos 2018-2019

\begin{tabular}{|l|l|l|l|l|}
\hline \multicolumn{1}{|c|}{ Empresa } & \multicolumn{1}{c|}{ País } & \multicolumn{1}{c|}{ Sector } & \multicolumn{1}{c|}{2018} & \multicolumn{1}{c|}{2019} \\
\hline Compañía Minera Lafayette S.A. & Perú & Minería & GRI Standards & GRI Standards \\
\hline GLM" SERV. HDPE SAC & Perú & Minería & GRI Standards & GRI Standards \\
\hline Roca Fuerte & Perú & Minería & & GRI Standards \\
\hline Sifalmin & Perú & Minería & & GRI Standards \\
\hline Sociedad Minera Cerro Verde SAA & Perú & Minería & & GRI Standards \\
\hline Sierra Gorda SCM & Chile & Minería & GRI Standards & \\
\hline Aela Energía & Chile & Energía & & GRI Standards \\
\hline Lipigas & Chile & Energía & GRI Standards & GRI Standards \\
\hline BAUM INGENIEROS EIRL & Perú & Energía & GRI Standards & \\
\hline Construredes SAC & Perú & Energía & GRI Standards & GRI Standards \\
\hline CREATECH INGENIERIA Y PROYECTOS SAC & Perú & Energía & GRI Standards & GRI Standards \\
\hline DAYRO CONTRATISTAS GENERALES S. R. L & Perú & Energía & GRI Standards & \\
\hline EGESUR & Perú & Energía & GRI Standards & \\
\hline Genersol & Perú & Energía & GRI Standards & \\
\hline Grupo INGESER & Perú & Energía & & GRI Standards \\
\hline HMV & Perú & Energía & GRI Standards & \\
\hline LARI & Perú & Energía & & GRI Standards \\
\hline OCA GLOBAL & Perú & Energía & & GRI Standards \\
\hline PERT INGENIEROS ASOCIADOS SAC & Perú & Energía & & GRI Standards \\
\hline QUANTA SERVICES PERU SAC & Perú & Energía & GRI Standards & \\
\hline RDR Consultores y Contratistas & Perú & Energía & GRI Standards & \\
\hline SIA INGENIERIA ESPECIALIZADA & Perú & Energía & & GRI Standards \\
\hline TURBOGENERADORES DEL PERÚ SAC & Perú & Energía & & GRI Standards \\
\hline
\end{tabular}

Fuente: Elaboración propia.

Fecha de recepción: 26 de setiembre de 2019

Fecha de aceptación: 25 de octubre de 2020 Correspondencia: karina.pocomucha@udep.edu.pe cecilia.venegas@udep.edu.pe 